\title{
Fluorescent Nuclear Track Detectors as a Tool for Ion-Beam Therapy Research
}

\author{
Greilich, S. ${ }^{a, *}$ Osinga, J.-M. ${ }^{a}$, Niklas, M. ${ }^{a}$, Lauer, F.M. ${ }^{a}$, Klimpki, G. ${ }^{a}$, \\ Bestvater, F. ${ }^{\mathrm{b}}$, Bartz, J.A. ${ }^{\mathrm{c}, \mathrm{d}}$, Akselrod, M.S. ${ }^{\mathrm{c}}$, Jäkel, O. ${ }^{\mathrm{a}, \mathrm{e}, \mathrm{f}}$ \\ ${ }^{a}$ Department of Medical Physics in Radiation Oncology, German Cancer Research \\ Center (DKFZ), Im Neuenheimer Feld 280, D-69120 Heidelberg, Germany \\ ${ }^{b}$ Light Microscopy Facility, German Cancer Research Center (DKFZ), Im Neuenheimer \\ Feld 280, D-69120 Heidelberg, Germany \\ ${ }^{c}$ Landauer Inc., 723 1/2 Eastgate, Stillwater, OK 74074, USA \\ ${ }^{d}$ Oklahoma State University, 145 Physical Sciences Bldg, Stillwater, OK 74078, USA \\ ${ }^{e}$ Heidelberg Ion-Beam Therapy Center, Im Neuenheimer Feld 450, D-69120 Heidelberg, \\ Germany \\ ${ }^{f}$ Radiation Oncology, University Hospital of Heidelberg, Im Neuenheimer Feld 400, \\ D-69120 Heidelberg, Germany
}

\begin{abstract}
Fluorescent nuclear track detectors based on $\mathrm{Al}_{2} \mathrm{O}_{3}: \mathrm{C}, \mathrm{Mg}$ with their excellent efficiency for detection of heavy charged particles and full 3D information from laser scanning microscopy allow a multitude of issues related to ionbeam cancer therapy to be tackled. A recently established read out protocol enables the utilization of a commercial microscope similar to those available in many life-science environments. This contribution illustrates the approach, its potential and limitations, as well as applications in clinical ion beams.
\end{abstract}

Keywords: luminescent dosimetry, aluminum oxide, confocal microscopy, radiation imaging

\section{Introduction}

Ion beam cancer therapy (IBCT) using swift heavy charged particles (HCPs), such as protons or ions, shows substantial benefits for the treatment of malignant tumors when compared to standard modalities such as

\footnotetext{
${ }^{*}$ Corresponding author, Tel: +49-(0)6221-42-2632, Fax: +49-(0)6221-42-2665

Email address: s.greilich@dkfz.de (Greilich, S.)
} 
MV X-rays or electrons. IBCT's enhanced relative biological efficiency is due to very localized energy depostion of particles with high linear energy transfer (LET) in nano- or micrometer size volumes. The well-known inverse depth dose curve yields superior dose conformity and thus steep gradients of dose or fluence, respectively, on millimeter scale. A better understanding of the fundamental physical and radiobiological mechanisms as well as practical applications in IBCT therefore require new dosimetry systems that work accurately at these small scales.

Fluorescent nuclear track detector (FNTD) technology based on $\mathrm{Al}_{2} \mathrm{O}_{3}: \mathrm{C}, \mathrm{Mg}$ single crystals and non-destructive read out by fluorescent confocal laser scanning microscopy (CLSM) can provide precise three-dimensional information on particle trajectories and high-resolution energy deposition patterns (Akselrod and Sykora, 2011). In contrast to $\mathrm{Al}_{2} \mathrm{O}_{3}: \mathrm{C}$ used in thermally and optically stimulated luminescence dosimetry, Mg-doped $\mathrm{Al}_{2} \mathrm{O}_{3}$ crystals have green coloration and contain high concentrations of $\mathrm{F}_{2}^{2+}(2 \mathrm{Mg})$ aggregate defects, responsible for the blue absorption band. These color centers undergo radiochromic transformation when crystals are exposed to ionizing radiation by capturing an electron and converting into a three-electron $\mathrm{F}_{2}^{+}(2 \mathrm{Mg})$ color center. When excited around $620 \mathrm{~nm}$, these new radiation-induced color centers exhibit a high quantum-yield intra-center fluorescence at $750 \mathrm{~nm}$ with a short lifetime of $75 \pm 5$ ns (Sanyal and Akselrod, 2005; Sykora and Akselrod, 2010). FNTDs are bio-compatible, chemically inert, and the recorded information is thermally and temporally stable. Detectors allow for multiple non-destructive read outs and do not require chemical post-processing, which makes them an attractive candidate for research in ion-beam cancer therapy.

Up to now, FNTDs have been read using dedicated custom-built imaging systems designed by Landauer Inc., mainly because commercial CLSMs could not provide sufficient sensitivity for the near-infrared (NIR) fluorescence. Recently, a new generation of CLSMs has emerged equipped with avalanche photo diodes (APDs) that replace the conventional photomultiplier tubes (PMT) for low-light applications. APDs are utilized today in many areas of life sciences and highly sensitive to NIR outperforming PMT and GaAsP detectors. In this contribution, we describe how latest generation CLSMs allows one to obtain high-resolution, high-contrast images from FNTDs (Fig. $1)$. 


\section{Method}

\subsection{Microscope}

We found that the Zeiss LSM710 ConfoCor 3 inverted CLSM along with its ZEN software (version 2009) is suitable for the read out of $\mathrm{Al}_{2} \mathrm{O}_{3}: \mathrm{C}, \mathrm{Mg}$ crystals. Besides three PMTs (one for transmitted light), it features two fiber-coupled, actively quenched APDs operated in photon counting (Geiger) mode.

\subsubsection{APD characteristics and settings}

The APDs used in the ConfoCor 3 module are produced by Perkin Elmer and surpass PMTs in their quantum efficiency to detect fluorescence around $750 \mathrm{~nm}$. According to the manufacturer, the APD quantum efficiency in the visual wavelength range is $40-75 \%$, the dark count rate $\eta_{\mathrm{DC}}^{\mathrm{APD}} \leq 250 \mathrm{~Hz}$, dead time $40 \mathrm{~ns}$, pulse width $15 \mathrm{~ns}$, after-pulse height is at $0.5 \%$ of the primary pulse for the duration of 100-500 ns. To protect the APDs against overload, the photon counting rate is limited to $20 \mathrm{MHz}$. If higher rates occur, APDs are switched off and subsequent pixels in the acquired image will be fully saturated. Due to statistical overlap of incoming photons in time and the Geiger mode of operation with finite dead time, the APDs show deviation from linearity at count rates above $1 \mathrm{MHz}$ and reach saturation close to the $20 \mathrm{MHz}$ limit (Fig. 2).

The microscope's ZEN software offers two user-defined transformation of APD data, 'digital gain' $g$ and 'digital offset'. These mode settings do not have any effect on actual photon counts $N$ but merely change the grey scale and histogram representation of pixel intensities.

\subsubsection{Lightpath, optical filters, and detector}

As an excitation source of the radiochromically transformed color centers we selected the $633 \mathrm{~nm}$ helium-neon laser (nominal output power $5 \mathrm{~mW}$, $100 \mu \mathrm{W}$ at the sample). An appropriate main beam splitter (MBS488/561/633) and a single longpass emission filter (LP655) in front of the APD were used for signal separation.

\subsubsection{Objective lenses}

We employed three objective lenses different in numerical aperture (NA), field of view and appropriate immersion medium (Tab. 1). Low-autofluorescent Zeiss Immersol ${ }^{\mathrm{TM}} 518 \mathrm{~F}$ was used in case of oil immersion objective lenses. Its refractive index $\hat{n}$ at $633 \mathrm{~nm}$ is $1.51\left(\hat{n}=1.77\right.$ for $\left.\mathrm{Al}_{2} \mathrm{O}_{3}\right)$. 
The ratio of average fluorescence intensities of the images in Fig. 3 is approx. 1:0.35:0.12 $(10 \times / 0.3 \mathrm{NA}, 40 \times / 1.4 \mathrm{NA}, 63 \times / 1.45 \mathrm{NA})$. Due to the ubiquitous high background of FNTDs (see 2.3.1), lenses with smaller NA deliver higher intensities but also significantly lower image resolution and contrast. The ratio of fluorescence signal in the track core and the unirradiated surrounding area was 1.5 for the $40 \times / 1$.4NA lens and 2.3 for $63 \times / 1.45 \mathrm{NA}$. This also explains why conventional epifluorescence microscopy is hardly successful with FNTDs.

We commonly set the pinhole diameter $d_{\mathrm{p}}$ to 1 Airy unit (AU) for optimal trade-off between resolution and image contrast and brightness. A further reduction increases contrast but at the expense of fluorescence intensity dropping significantly, e.g. by $75 \%$ for $0.5 \mathrm{AU}$.

\subsection{Samples}

In all experiments, we used $\mathrm{Al}_{2} \mathrm{O}_{3}: \mathrm{C}, \mathrm{Mg}$ single crystals produced by Landauer Inc. in the form of rectangular plates having dimensions of $4 \times 8 \times$ $0.5 \mathrm{~mm}^{3}$ or $4 \times 6 \times 0.5 \mathrm{~mm}^{3}$, with the long side cut along the optical $c$-axis and with one large surface polished to optical quality (Tab. 2).

\subsubsection{Sample handling}

Glass bottom microwell dishes from MatTek Corp., Ashland, MA, USA (Part No. P35G-1.5-20C) with 160 - $190 \mu \mathrm{m}$ thick cover slips $(\hat{n}=1.52$ at $633 \mathrm{~nm}$ ) were employed to hold the samples (Fig. 1, right). They provide easy sample changing, positioning and rotation while avoiding contamination of the detector with immersion oil. The coupling and light absorption when using immersion oil only between the lens and the dish was found more favorable than using oil directly between FNTD and the lens.

\subsubsection{Sample orientation}

$\mathrm{Al}_{2} \mathrm{O}_{3}: \mathrm{C}, \mathrm{Mg}$ single crystals have a slightly distorted hexagonal closed packed structure with the main optical $c$-axis aligned along the longer crystal side (Sanyal and Akselrod, 2005). For efficient excitation laser light absorption, the polarization direction of the laser light was aligned with the optical $c$-axis $(c|| E)$ of the crystal. The read out was done from the polished side of the crystal, i.e. face down in the inverted setup. 


\subsection{Image acquisition and signal processing}

During image acquisition the focused excitation laser is scanned laterally in $x$ and $y$-directions (forming one 'field') by two galvanometer mirrors. Multiple images in depth $(z)$ were acquired by moving the focal plane of the objective lens inside the crystal using the piezo-electric actuator of the translation table and thus the sample relative to the lens.

The ZEN software lets the operator control the field of view size $l_{\mathrm{x}}$ and $l_{\mathrm{y}}$, the number of sample points (the 'pixels') $n_{\mathrm{x}}$ and $n_{\mathrm{y}}$ within the field and the scan speed via a dwell time per pixel $\tau$ (other vendors rather refer to the scan frequency $T$ ). Also, the number of optical slices in depth $n_{\mathrm{z}}$ and their interval $d_{z}$ can be set. The image pixel density $d=l / n$ must not be confused with the actual microscope resolution defined by the system's point-spread function and its lateral and axial dimensions $\sigma_{\mathrm{x}, \mathrm{y}}$ and $\sigma_{\mathrm{z}}$, respectively (Tab. 1 ). The field of view size $l_{x}$ and $l_{y}$ can also be indirectly varied via a 'zoom' dialog, as well as the field location and its rotation.

The software reports the APD photon counts $N$ per pixel for a sum of $R$ of repetitive line or frame scans. Since Poisson-distributed shot noise yields a relative uncertainty of $1 / \sqrt{N}$ per pixel and is the major source of image noise, an increase in $\tau$ and $R$ increase $N$ and thus the image quality. However, ZEN is not able to go beyond $N_{\max }=65535$ due to the limitation to 16 bit (which was always used for maximum bit depth). In theory, this could be partly circumvented, e.g. by using averaging during rescans. But since ZEN also truncates averaging results to integer numbers this yields a significant reduction in dynamic range. Also, large values of $\tau$ and $R$ increase the overall acquisition time.

To compare APD signals for various settings of $\tau$ and $R$, the count rate per pixel can be computed by

$$
\eta=\frac{N}{\tau \cdot R} .
$$

Typical values of $\tau$ for the LSM710 are in the range from $1.3 \mu$ s to $177 \mu \mathrm{s}$, $R$ can be set to any number greater or equal 1 (single scan). In order to adjust the maximum count rate of the individual sample's fluorescence signal not to exceed $\eta_{\max }=20 \mathrm{MHz}$, the relative power $p$ of the excitation laser can be attenuated by an acousto-optical tunable filter (AOTF) from $100 \%$ to $0.2 \%$ of the output power. To still keep comparability of measurements, we eliminate the additional influence of the relative laser power $p$ by defining the 'adjusted count rate': 


$$
\eta^{\mathrm{adj}}=\frac{\eta-\eta_{\mathrm{DC}}}{p}
$$

where $\eta_{\text {DC }}$ refers to the total dark count rate from both the APD and the residual room light. The maximum acceptable adjusted count rate is hence $\eta_{\max }^{\text {adj }}=10 \mathrm{GHz}$. In practice, avoiding APD overload is achieved by monitoring the online representation of photon counts $N_{\text {grey }}$ in the histogram feature of the ZEN software. Although irrelevant for the eventually recorded data of $N$, we have here to consider the digital gain $g$ :

$$
N_{\text {grey }} \stackrel{!}{\leq} \frac{\tau \cdot R \cdot p}{g} \cdot 20 \mathrm{MHz}
$$

Since $N_{\text {grey }}$ is truncated to integer values it is beneficial to set $g$ to the lowest possible value of 0.2 .

\subsubsection{Background and unwanted sources of signal variability}

FNTDs are known to exhibit fluorescence even before the irradiation and from the unirradiated part of the image. This 'background' varies significantly both within a single crystal and from crystal to crystal $\left(\eta_{\mathrm{bkg}}^{\text {adj }}=\right.$ $400-600 \mathrm{kHz}$, Tab. 2). The variation of background fluorescent signal on small scales of approximately $1 \mu \mathrm{m}$ are up to $10 \%$ (Fig. 4) while over larger distances of several tens of micrometers or between samples differences up to a factor 2 were found. We used the 'Mosaic' group's plugin (Cardinale, 2010) for the ImageJ software (see section 2.3.3) which very effectively removes large scale background. However, the small scale variations have to be considered as a medium noise and are the dominant factor for the lower dose limit of the technique. Similar limitation imposed by the background fluorescence of $\mathrm{Al}_{2} \mathrm{O}_{3}: \mathrm{C}, \mathrm{Mg}$ were demonstrated in the case of radiophotoluminescent dosimetry application of this material (Eller et al., 2013; Ahmed et al., 2013).

We did not find hints of any significant noise influence such as laser variability, vibration or electro-magnetic interference. Also, the adjusted dark count rate of the APDs $\eta_{\mathrm{DC}}^{\mathrm{APD}, \mathrm{adj}}=250 \mathrm{~Hz} / p$ is negligible. Similar, the typical adjusted count rate due to residual room light under measurement conditions $\left(\eta_{\mathrm{DC}}^{\text {room }, \text { adj }} \approx 3.5 \mathrm{kHz} / p\right.$ ) is usually insignificant - with the important exception of low laser power settings $(p \lesssim 2 \%)$.

Dust, autofluorescent particles and imperfections on the FNTD surface may have a significant impact on the count rate. Although some of these 
artifacts can be eliminated or corrected in post-processing they should be avoided during any measurement as much as possible. For example, by using air coupling (instead of immersion oil) between a crystal and immaculate glass-bottom dishes one avoids the negative effect of bubble formation in the immersion fluid and reduces the number of fluorescent objects on the crystal surface as compared to direct mounting of FNTDs on cover slips. Careful cleaning of the crystal surface using an ultrasonic bath reduces the number of unwanted image artifacts.

Within the FNTD volume, spinel cubical inclusions are visible as distinct dark spots with both significantly reduced background and radiation induced fluorescence (Fig. 3, left image).

\subsubsection{Signal-to-noise ratio}

The signal-to-noise ratio (SNR) $S$ is a more appropriate measure of image quality for FNTD application than the relative counting error $1 / \sqrt{N}$. In its simplest form the SNR can be defined as the difference of counts in a track's core and background counts divided by the square root of the sum of their respective Poisson errors:

$$
S=\frac{N_{\text {core }}-N_{\text {bkg }}}{\sqrt{N_{\text {core }}+N_{\text {bkg }}}} .
$$

A more detailed analysis would have to include the size of tracks and spatial patterns. $S$ in Eq. 4 depends on the absolute counts and thus on the microscope settings $\tau, R$, and $p$. Therefore we define an 'SNR factor' $\hat{S}$ by

$$
\hat{S}=\frac{N_{\text {core }} / \tau R p-N_{\mathrm{bkg}} / \tau R p}{\sqrt{N_{\text {core }} / \tau R p+N_{\mathrm{bkg}} / \tau R p}}=\frac{\eta_{\mathrm{core}}^{\mathrm{adj}}-\eta_{\mathrm{bkg}}^{\mathrm{adj}}}{\sqrt{\eta_{\mathrm{core}}^{\mathrm{adj}}+\eta_{\mathrm{bkg}}^{\mathrm{adj}}}} .
$$

The SNR $S$ can be estimated from the sample specific $\hat{S}$ for any read out setting

$$
S=\hat{S} \cdot \sqrt{\tau R p}
$$

\subsubsection{Image processing}

We found that the correct display of images acquired by the APDs was severely hampered by several restrictions within the ZEN software version used. However, image data containing the APD counts $N$ and stored in Zeiss' 
LSM5 file format can seamlessly be processed by ImageJ, a free Java program developed by Wayne Rasband (Rasband, 1997-2011; Abramoff et al., 2004). We used version 1.43u and later together with the 'Input-Output/LSM_reader' plugin. More extensive, scripted data processing was done within the $\mathrm{R}$ software (R Development Core Team, 2010) using a dedicated custom developed package ('FNTD') which again employs ImageJ plugins.

\subsection{Sensitivity}

\subsubsection{Response to photon radiation}

Fig. 5 summarizes the results from 13 samples irradiated with photon doses from $0.01 \mathrm{~Gy}$ to $10 \mathrm{kGy}$. The saturation dose of $\mathrm{Al}_{2} \mathrm{O}_{3}: \mathrm{C}, \mathrm{Mg}$ was found to be $D_{0}=83.3 \pm 7.1 \mathrm{~Gy}$ and thus comparable to values reported for $\mathrm{Al}_{2} \mathrm{O}_{3}$ : C (Yukihara et al., 2004). The maximum adjusted count rate measured were in the order of $\eta_{\max }^{\text {adj }} \approx 400 \mathrm{MHz}$. The response of the FNTD decreases again for very large doses $(\geq 1 \mathrm{kGy})$. The samples were also reported to be darkened with increasing dose immediately after irradiation but this effect disappeared until the time of read out two days later (N. Hünemohr, personal communication).

The lower panel of Fig. 5 shows exemplary images from which the gamma dose response curve was derived. Single secondary electrons tracks can clearly be identified and their density visibly increases with dose. This points to a strong granularity of photon radiation on a micrometer scale and questions the assumption that photons deposit energy in matter relatively homogeneously. Instead, similar to HCP tracks (but very different in LET) photon induced electron tracks can be considered as a discrete entity with specific efficiency. The linear part of the photon response curve is due to an increase in fluence until track overlap becomes significant causing onset of saturation.

\subsubsection{Microscope sensitivity}

The data in Fig. 5 allows one to calculate the photon sensitivity factor for the adjusted count rate in the linear region of the dose dependence equal to $3.58 \mathrm{MHz} / \mathrm{Gy} / p$. If we consider the laser power and the lateral area of the point-spread function (for the $63 \times / 1.45 \mathrm{NA}$ lens, Tab. 1), we get an estimate for the microscope sensitivity of $7.9 \cdot 10^{-3} \mathrm{~Hz} \cdot \mathrm{m}^{2} /(\mathrm{Gy} \cdot \mathrm{W})$. This is surely a very rough measure which nevertheless will allow us to compare the performance of the LSM710 (for similar FNTD samples) to other systems. 


\subsubsection{HCP radiation}

The FNTD background fluorescence values $\eta_{\mathrm{bkg}}^{\mathrm{adj}}$ (Tab. 2) correspond to apparent photon doses in the order of 0.1 to 0.2 Gy. For the track cores, the radiation doses measured by the fluorescent intensities $\eta_{\text {core }}^{\text {adj }}$ (Tab. 2, 0.4 Gy to $4.7 \mathrm{~Gy}$ for protons and carbon ions, 14 and $55 \mathrm{~Gy}$ for Fe and Xe, resp.) are clearly underestimated due to the following reasons:

(a) Averaging of the track core fluorescence signal due to the much larger point-spread function of the imaging system.

(b) Saturation of fluorescence due to the limited number of color centers available in the actual track core where very high energy densities (corresponding to MGy) can occur.

(c) Saturation of APDs at high count rate (this is the case of the ${ }^{56} \mathrm{Fe}$ ion beam irradiated sample).

To avoid the saturation of the APD as in the latter case, simple laser power reduction is needed which cannot however change the saturation of the color centers in the track core at high LETs.

The measured spatial distribution of energy deposited in the HCP track could be further improved (Fig. 6) by using super-resolution microscopy techniques (optical nanoscopy) such as simulated emission depletion (Hell, 2007).

We can conclude from the measurements presented that despite limitations of the present ConfoCor 3 APDs operated in Geiger mode, our approach covers the entire dynamic range of fluorescence intensity by present FNTD technology. Most of our present applications in IBCT are, however, 'binary', i.e. they assess the presence, location or direction of a track rather than its quantitative shape. From the SNRfactor given in Tab. 2 and Eq. 6 it can be found that the LSM710 ConfoCor3 provides sufficient track signatures even for fast $(220 \mathrm{MeV})$ protons and hence for all other particles and energies met in IBCT.

\section{Conclusion}

We used a commercial CLSM equipped with APDs to successfully read out $\mathrm{Al}_{2} \mathrm{O}_{3}$ :C,Mg-based fluorescent nuclear track detectors with fully adequate performace for clinical HCP beams. We believe this allows for an easy implementation of the FNTD technology in life sciences where similar CLSMs become increasingly available. Our special focus is hereby on ion beam radiotherapy and radiobiology research where the described approach 
has been already used for high-accuracy track counting and fluence assessment (Osinga et al., 2013), the measurement of ion ranges and hence energy, as a feasibility study of future in-vivo use of FNTDs (Klimpki et al., 2013), and the three dimensional visualization of particle trajectories and energy deposition patterns (Niklas et al., 2013a). The latter work demonstrated a new approach for colocalization radiobiology studies where HCP trajectories propagating through the cell culture grown on top of the FNTDs can be spatially correlated with the DNA double-strand breaks (Niklas et al., 2013b). More general, FNTDs can widely serve to characterize radiation fields used in IBCT. Future applications could be the assessment of microdosimetric quantities or single track spectroscopy.

\section{Acknowledgments}

We are deeply indebted to numerous colleagues for their enthusiasm and continuous support for our project: Thomas Underwood from Landauer for FNTD sample preparation, Manuela Brom from DKFZ light microscopy facility for her technical assistance, Stefan Brons from HIT, Roland Repnow and Manfred König from MPI-K, Rochus Herrmann from Aarhus University, Jakob Helt-Hansen and Claus E. Andersen from DTU Nutech, Roskilde, and Andrea Schwahofer, Nora Hünemohr and Roman Martel from DKFZ for their help with FNTD irradiations.

Abramoff, M.D., Magelhaes, P.J., Ram, S.J., 2004. Image processing with ImageJ. Biophotonics Int 11, 36-42.

Ahmed, M., Eller, S., Bartz, J., Akselrod, M., Denis, G., Yukihara, E., 2013. Comparison between different readout approaches for aluminum oxide radiophotoluminescent crystals. this issue .

Akselrod, M.S., Sykora, G.J., 2011. Fluorescent nuclear track detector technology - a new way to do passive solid state dosimetry. Radiation Measurements 46, 1671-1679.

Cardinale, J., 2010. Histogram-based background subtractor for ImageJ. Technical Report. ETH Zurich.

Eller, S., Ahmed, M., Bartz, J., Akselrod, M., Denis, G., Yukihara, E., 2013. Photoluminescence properties of aluminum oxide radiophotoluminescent crystals. this issue . 
Hell, S.W., 2007. Far-field optical nanoscopy. Science 316, 1153-1158.

Klimpki, G., Osinga, J.M., Herrmann, R., Akselrod, M., Jäkel, O., Greilich, S., 2013. High-accuracy ion range measurements using fluorescent nuclear track detectors. this issue .

Niklas, M., Melzig, C., Abdollahi, A., Akselrod, M., Debus, J., Jäkel, O., Greilich, S., 2013a. A new method for analyzing energy deposition patterns of ion beams in cell. submitted to International Journal of Radiation Onkology * Biology * Physics .

Niklas, M., Melzig, C., Abdollahi, A., Bartz, J., Akselrod, M., Debus, J., Jäkel, O., Greilich, S., 2013b. FNTD-/cell-hybrid system - towards single track spectroscopy. this issue .

Osinga, J.M., Akselrod, M., Jäkel, O., Greilich, S., 2013. High-accuracy fluence determination in ion beams using fluorescent nuclear track detectors. this issue .

R Development Core Team, 2010. R: A Language and Environment for Statistical Computing. R Foundation for Statistical Computing. Vienna, Austria. URL: http://www.R-project.org. ISBN 3-900051-07-0.

Rasband, W.S., 1997-2011. Imagej. URL: http://rsb.info.nih.gov/ij/.

Sanyal, S., Akselrod, M.S., 2005. Anisotropy of optical absorption and fluorescence in $\mathrm{Al}_{2} \mathrm{O}_{3}$ : C,Mg crystals. Journal of Applied Physics 98, 033518-1 $-033518-12$.

Sykora, G.J., Akselrod, M.S., 2010. Photoluminescence study of photochromically and radiochromically transformed $\mathrm{Al}_{2} \mathrm{O}_{3}$ crystals used for fluorescent nuclear track detectors. Radiation Measurements 45, 631-634.

Yukihara, E., Whitley, V., McKeever, S., Akselrod, A.E., Akselrod, M., 2004. Effect of high-dose irradiation on the optically stimulated luminescence of $\mathrm{Al}_{2} \mathrm{O}_{3}$ :C. Radiation Measurements 38, 317-330. 

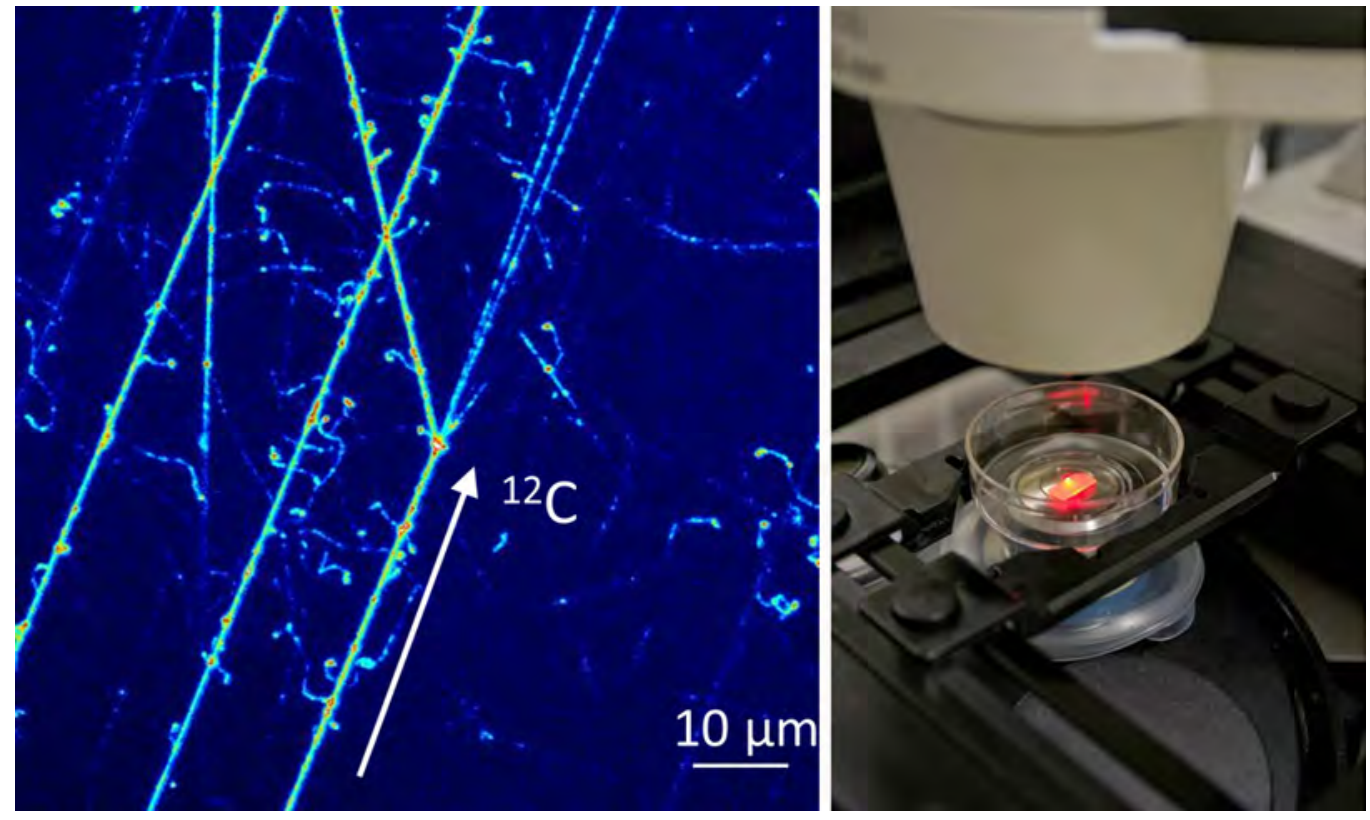

Figure 1: FNTD image (left) obtained using the Zeiss LSM710 ConfoCor 3 laser scanning confocal microscope (right) equipped with avalanche photo diodes. Carbon ions with a kinetic energy of $270 \mathrm{MeV} / u$ were incident parallel to the images crystal plane. The images is a maximum intensity projection of 13 images from different optical slices, resp. Beside the primary ion tracks, secondary electron tracks (curly features), a fragmentation event (center of left panel) and light fragments tracks (thin straight lines) are clearly visible. 


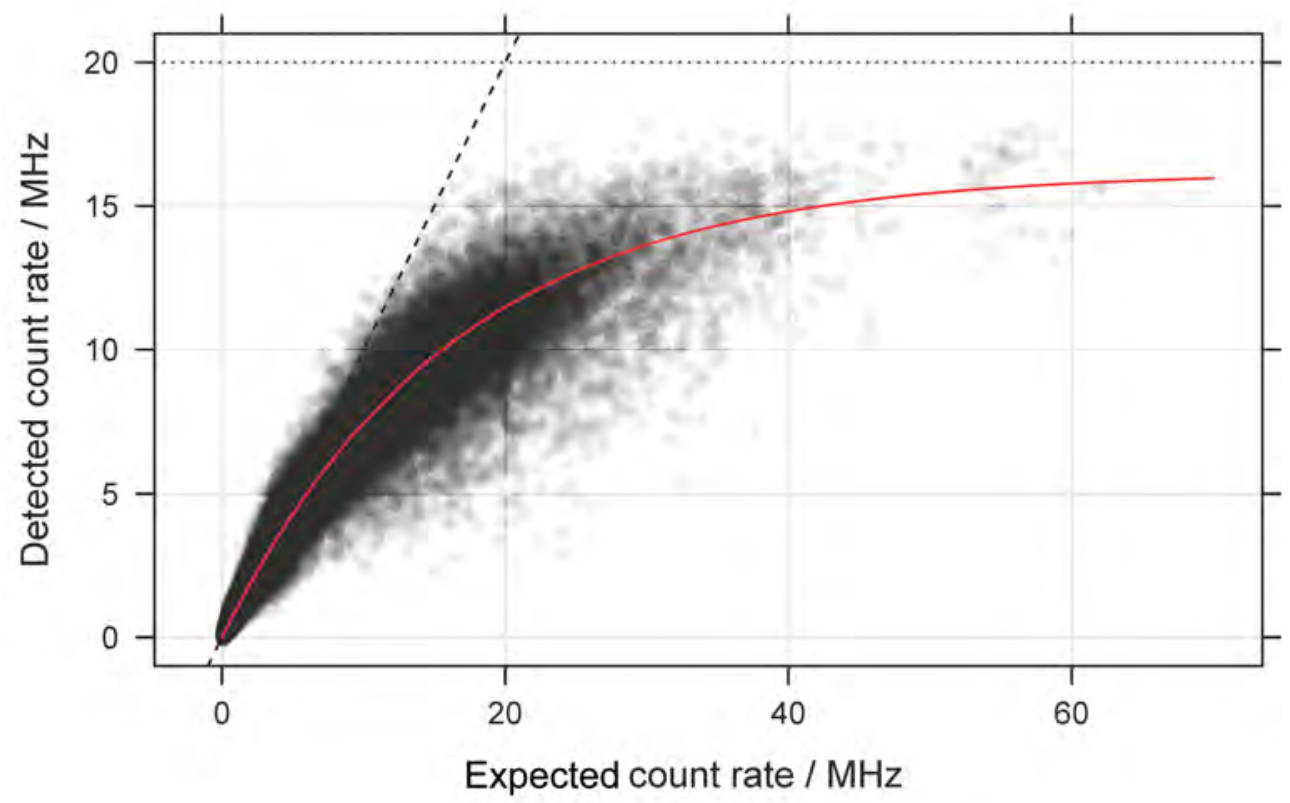

Figure 2: Detected APD count rate vs. expected photon counting rate estimated from laser power. $633 \mathrm{~nm} \mathrm{He}-\mathrm{Ne}$ laser light was reflected from a glass slide at sample position, and a series of images was taken at nine laser power settings from $0.2 \%$ to $100 \%$. A model using an inverted exponential function was fitted to the scatter-plot data obtained from the images with a wide range of intensities. The dashed line indicated a 1:1 identity, the dotted line is the APD saturation count rate $\eta_{\max }$ as provided by the vendor, and the solid red line is the model. The expected count rate was assumed to be linear with laser power. The average saturation count rate $\eta_{0}$ was estimated to be $16.2 \mathrm{MHz}(\sigma=1.4 \mathrm{MHz}$, $\left.\sigma_{\mu}=0.02 \mathrm{MHz}\right)$. 


\begin{tabular}{l|ccccc} 
objective lens & $\sigma_{\mathrm{x}, \mathrm{y}} / \mu \mathrm{m}$ & $\sigma_{\mathrm{z}} / \mu \mathrm{m}$ & $l / \mu \mathrm{m}\left(n^{\mathrm{opt}}\right)$ & $d_{\mathrm{x}, \mathrm{y}}^{\mathrm{opt}} / \mu \mathrm{m}$ & $d_{\mathrm{z}}^{\mathrm{opt}} / \mu \mathrm{m}$ \\
\hline $10 \times / 0.3 \mathrm{NA}$ & 0.94 & 12.8 & $849.7(1828)$ & 0.47 & 6.4 \\
$40 \times / 1.4$ NA Oil & 0.22 & 1.0 & $214.4(1980)$ & 0.11 & 0.48 \\
$63 \times / 1.45$ NA Oil & 0.20 & 0.8 & $134.9(1352)$ & 0.10 & 0.41
\end{tabular}

Table 1: Characteristics of lenses used for FNTD read out. Zeiss' objective lens identification contains magnification, numerical aperture, and immersion liquid where applicable. The lateral size of the point-spread function (FWHM) $\sigma_{\mathrm{x}, \mathrm{y}}$ is given for an excitation wavelength $633 \mathrm{~nm}$, the axial size $\sigma_{\mathrm{z}}$ is estimated for 1 Airy unit (AU) of the detection pinhole diameter. The field of view size $l$ is given for a zoom parameter of 1.0. The sample density according to Nyquist's theorem $d_{\mathrm{xy}}^{\mathrm{opt}}=0.5 \cdot \sigma_{\mathrm{x}, \mathrm{y}}$ and optimal depth slice interval $d_{\mathrm{z}}^{\mathrm{opt}}=0.5 \cdot \sigma_{\mathrm{z}}$ were selected according to the manufacture's guidelines. No refractive index correction from glass $(\hat{n}=1.51)$ to sapphire $(\hat{n}=1.77)$ was applied. 

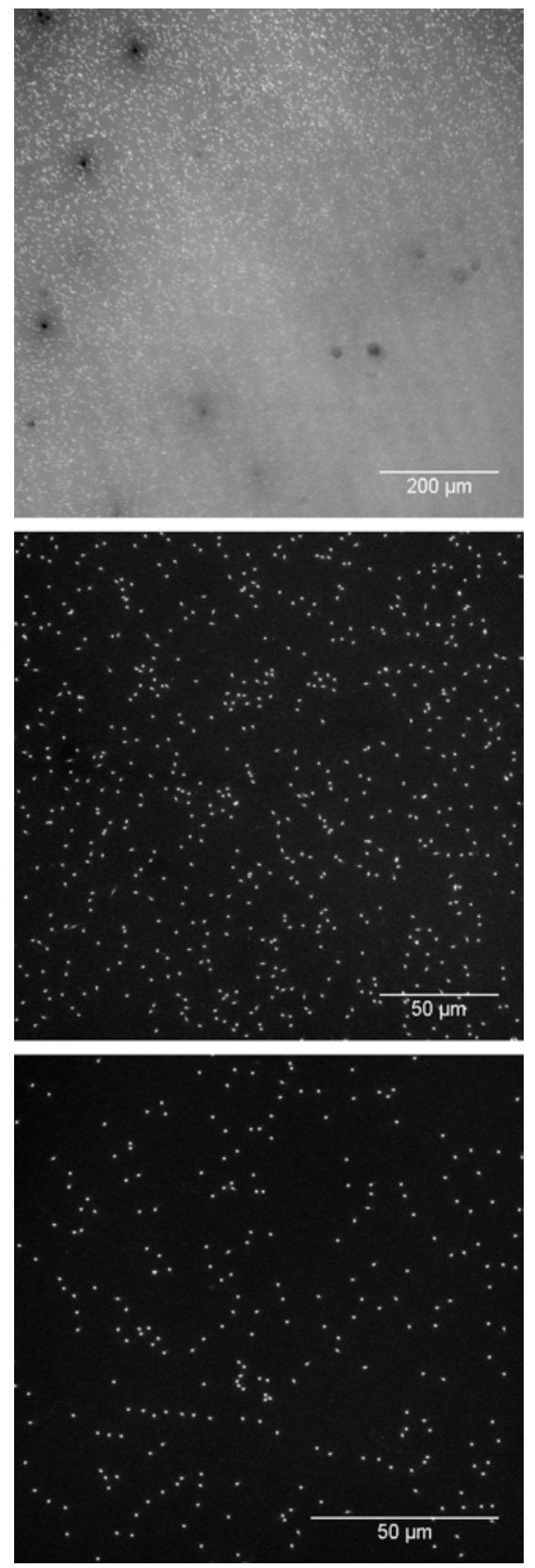

Figure 3: FNTD sample (sg35041) irradiated with 3 MeV protons impinging perpendicular to the image plane and read out with the three available objective lenses and a corresponding pinhole size of 1 Airy unit (AU, from top to bottom: $10 \times / 0.3 N A, 40 \times / 1.4 N A$, $63 \times / 1.45 \mathrm{NA})$. For the $10 \times / 0.3 \mathrm{NA}$ objective lens, the actually perpendicular tracks seem to lean towards the center of the image. This spherical abberations are less evident when using $40 \times / 1.4 N A$ and $63 \times / 1.45 N A$ lenses but can be seen when using maximum intensity projection of image stacks obtained at different depths. In the bottom panel, Al-Al spinel cubical inclusions are visible as darker spots. $15_{\text {All measurements were done under the same }}$ read out conditions $\left(d_{\mathrm{p}}=1 \mathrm{AU}, \tau=177.1 \mu \mathrm{s}, p=100 \%, R=4\right.$, for definition see section 2.3). 

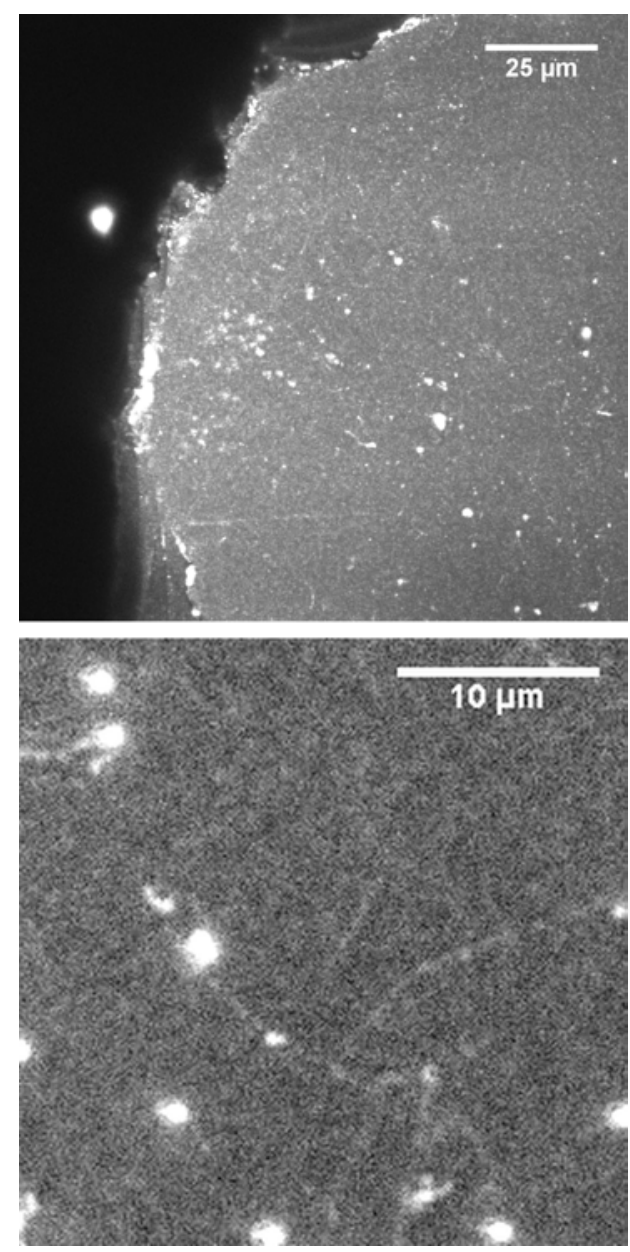

Figure 4: Fluorescence background of an unirradiated (upper) and irradiated (lower) samples. The background structure is stable during repeated read out. Thus, these variations cannot be attributed to variations in optics, laser intensity, detection efficiency and amplification noise. In the left panel residual fluorescent contaminations on the FNTD surface are seen. 

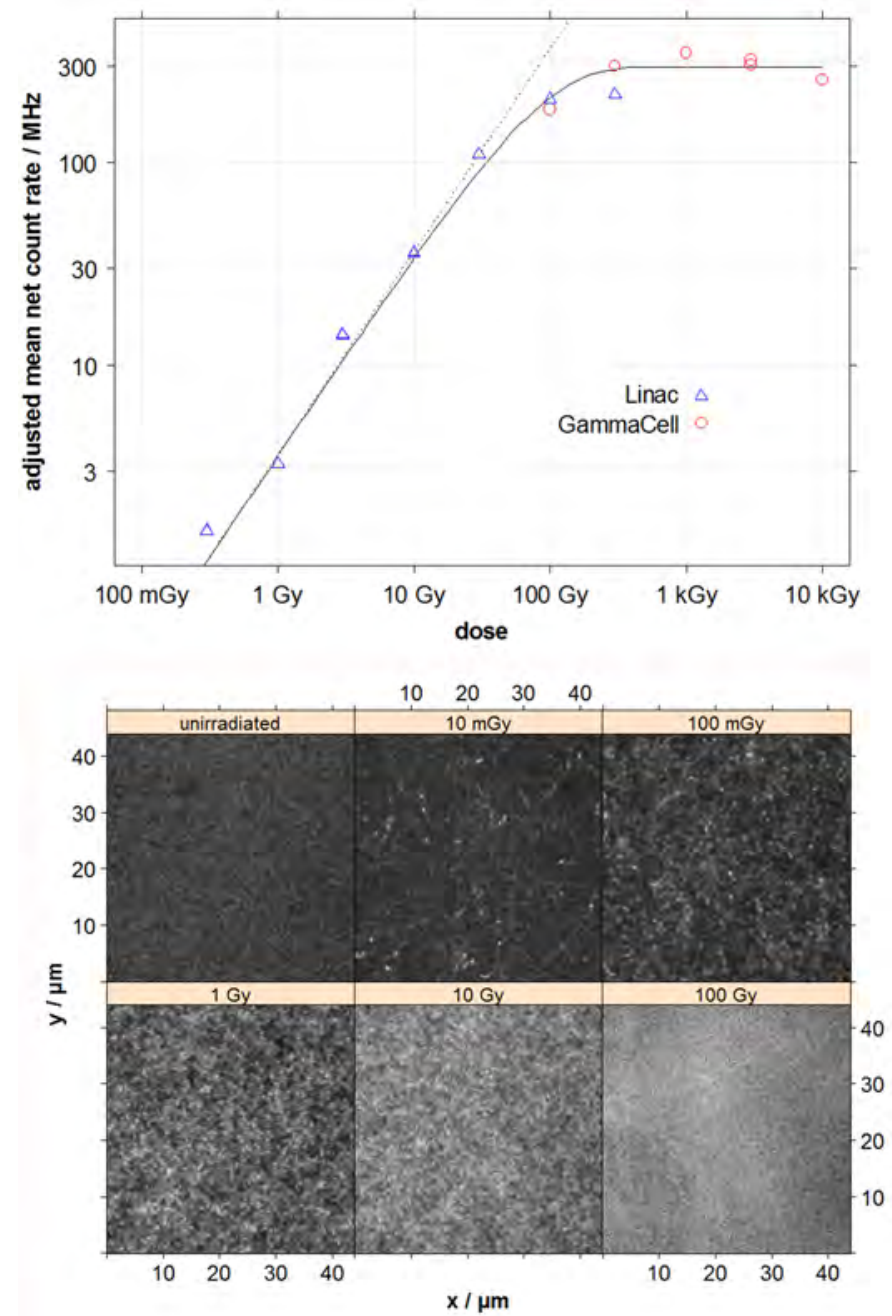

Figure 5: Upper panel: Adjusted count rate dependence on photon dose for FNTD irradiated with $6 \mathrm{MV} X$-rays (circles) and ${ }^{60} \mathrm{Co}$ (triangles). The dashed line shows a linear relation, the solid line is a fit of the data to the inverse exponential function. Samples below 0.3 Gy were excluded from the analysis due to the high influence of $\eta_{\mathrm{DC}}$ at the low laser power settings. Read out parameters were kept constant for all samples and adjusted to the highest measured signals found (63x objective, $\tau=12.7 \mu \mathrm{s}, p=1 \%, R=1 \ldots 16$, $\left.d_{\mathrm{p}}=1 A U\right)$. Lower panel: Zoomed images of FNTDs irradiated with increasing photon doses (field of view $l=44 \mu \mathrm{m}, n=376 \mathrm{px}$ ). The intensity was adjusted for each image individually. For the unirradiated sample, typical background variation on the $\mu \mathrm{m}$ scale $( \pm 5 \%)$ can be seen. For the 100 Gy sample, photon shot noise is dominant as all color centers in the crystal are saturated by radiochromic transformation. 


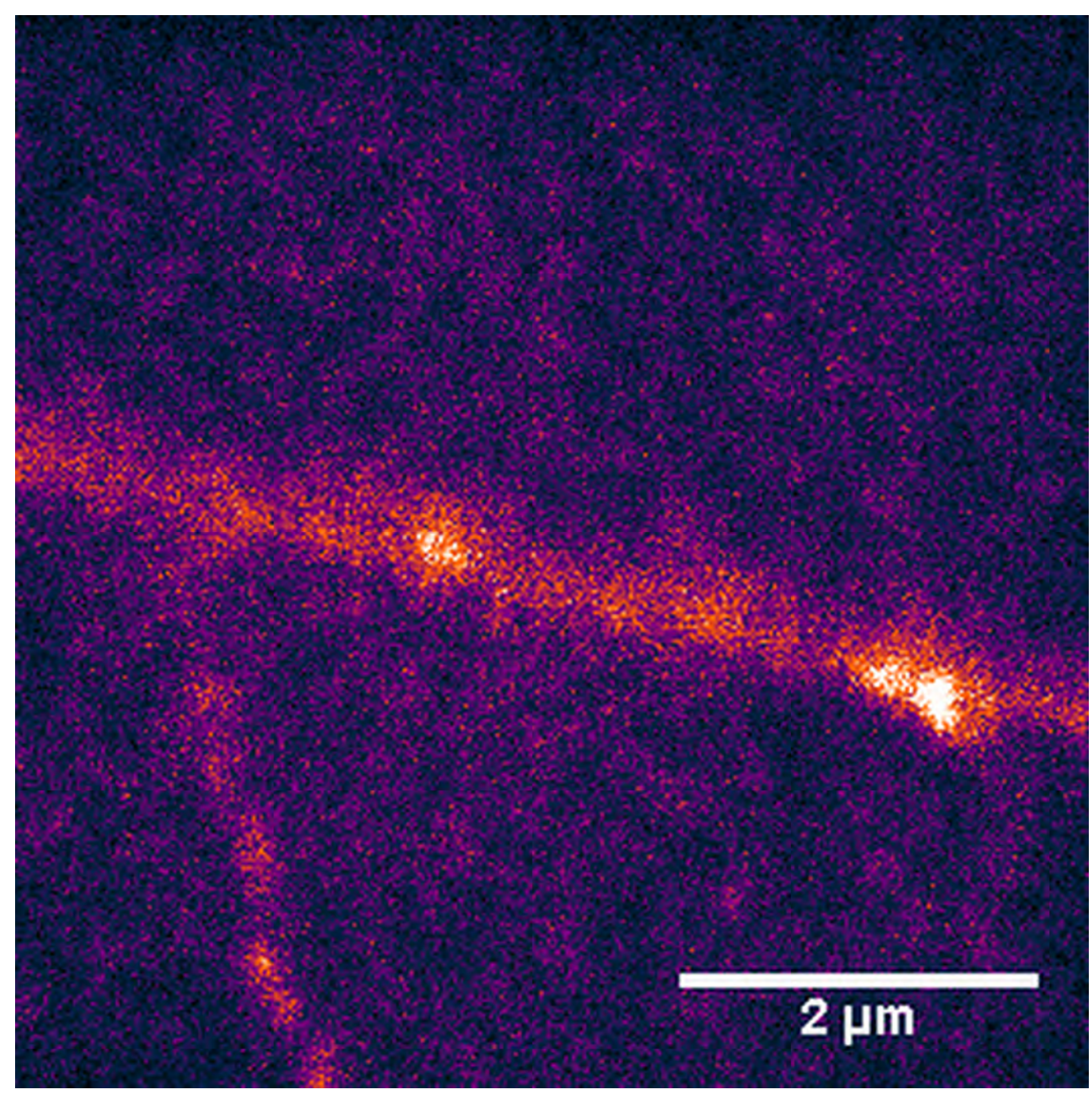

Figure 6: STED (STimulated Emission Depletion) image of a carbon ion track (Courtesy of M. Henrich, J. Engelhardt, Division of Optical Nanoscopy, DKFZ). The resolution is approx. $100 \mathrm{~nm}$. 
